\section{Redundant-target detection and processing capacity: The problem of positional preferences}

\author{
PAUL A. MULLIN, HOWARD E. EGETH, \\ and J. TOBY MORDKOFF \\ The Johns Hopkins University \\ Baltimore, Maryland
}

Although the capacity issue is fundamental to the understanding of human performance, there has been considerable difficulty in designing methodologies that distinguish between limited-capacity and unlimited-capacity processing models. The mathematical complexity of this enterprise has been discussed in a series of papers by Townsend and his colleagues (Snodgrass \& Townsend, 1980; Townsend, 1971, 1972, 1974). In these papers, Townsend demonstrated that models of various capacities can be made to mimic the predictions of one another. For example, Townsend notes that serial and parallel models are not strictly identifiable on the basis of the relationship between reaction time (RT) or accuracy and the number of elements in visual search tasks.

One task for which differential predictions are made by limited-capacity and unlimited-capacity processing models is the redundant-target detection task (e.g., Biederman \& Checkosky, 1970; Egeth, Folk, \& Mullin, in press; Mullin \& Egeth, in press; Snodgrass \& Townsend, 1980; van der Heijden, 1975; van der Heijden, La Heij, \& Boer, 1983). In this task, the number of simultaneously presented items is varied. On a given trial, only items assigned to a single response are displayed. Mean RTs are then compared to determined if there is a decrease in RT with increasing numbers of targets, an effect known as a redundancy gain (for related work with redundant targets using accuracy measures, see Eriksen, 1966, and Santee \& Egeth, 1982). Snodgrass and Townsend (1980) have shown that limited-capacity models (both serial and parallel) are incompatible with a redundancy gain, but a spatially parallel, self-terminating, unlimited-capacity model remains plausible if response latency is determined by the first input to finish processing, since one of several inputs will finish processing faster on average than the average processing time for a single input.

One difficulty with the use of the redundant-target detection task is that limited-capacity processing can result in an artifactual redundancy gain if subjects have preferences or differential abilities to process certain types of information (Biederman \& Checkosky, 1970). Although

This research was supported in part by Grant BNS-8420151 from the National Science Foundation and in part by Grant 87-0180 from the AIr Force Office of Scientific Research (87-0180). The authors would like to thank Charles Folk and Steve Yantus, among others, for helpful comments and discussion throughout this project. Reprint requests should be sent to Howard Egeth, Department of Psychology, The Johns Hopkıns University, Baltimore, MD 21218.
Biederman and Checkosky were concerned with stimulus dimensions such as size and brightness, their arguments are generalizable to spatial locations. Similarily, the arguments presented here concerning spatial locations apply equally well to stimulus dimensions.

Consider the situation that occurs when displays contain two stimulus locations and one of the positions is preferred. Trials on which a single stimulus is displayed in a location that is not preferred will be relatively slow on average compared with trials on which a single stimulus appears in a favored position. For redundant-target trials, however, stimuli are presented in both the favored and unfavored position on every trial. Therefore, there is no particular subset of slow RTs for this condition. On most redundant-target trials, the proportion of which is inversely related to the degree of overlap between the distributions of processing times for the favored and unfavored locations, RT will be solely determined by the efficiency of stimulus processing at the favored location. When the overall means from the single-target and redundant-target conditions are compared, the mean RT will be elevated for the single-target trials due to the inclusion of slow trials in which the stimulus appeared in the position that was not favored. Thus, this comparison results in an artifactual redundancy gain. A similar logic applies when the number of redundant targets presented is greater than two (see van der Heijden et al., 1983). Biederman and Checkosky (1970) and van der Heijden et al. (1983) considered one type of artifactual redundancy gain that might result if each subject were to consistently favor a stimulus location. They suggested useful analyses for indicating whether there is a redundancy gain greater than what is expected on the basis of consistently favored positions. We have suggested a new analysis, referred to as the fixed-favored-position indicator, that is useful for determining whether any position is consistently favored (Egeth, Folk, \& Mullin, in press; Mullin \& Egeth, in press).

An artifactual redundancy gain may also result if subjects randomly vary their preference for a position on a trial-by-trial basis. Van der Heijden et al. (1983) suggested an analysis for dealing with this situation. For simplicity, again suppose that there are two possible stimulus locations, one of which is favored on each trial. Van der Heijden et al. argued that on approximately half of the single-target trials, the target will be located in the favored position. On the other half of these trials, the target will be located in the position that is not favored, and a relatively slow response can be expected. The RTs for all of the single-target trials are ordered from fastest to slowest and then divided into two subsets of equal size (three subsets would be formed if there were three possible stimulus locations, on the assumption that the target would appear in the favored location on about one-third of the trials). The difference between the means of the 
fast and slow ordered subsets should reflect any additional variability introduced by the positional preferences. If the same procedure is applied to the redundant-target trials, the difference between the means for the fast and slow ordered subsets should only reflect random variation. The differences between the mean RTs of the single-target and redundant-target conditions are then calculated separately for the fast and slow ordered subsets. Van der Heijden et al. argued that if there is a significant redundancy gain for both the fast and slow subsets, but a larger gain for the slow subsets, then the data are consistent with a limited-capacity model operating in conjunction with randomly varying positional preferences. However, if the redundancy gain is equal for the fast and slow subsets, then the data are consistent with an unlimited-capacity model with no positional preferences.

Contrary to van der Heijden et al. (1983), we have concluded that the redundancy gain should be smaller for the fast subsets than for the slow subsets when processing $o c-$ curs in parallel with unlimited capacity. When the completion time for one of the inputs falls near the fast end of the distribution of processing times, the probability that the other input will finish faster by a large margin is small. Conversely, if one input finishes near the slow end of the distribution, then the other input might finish a great deal faster. In other words, there is a floor effect in the magnitude of the redundancy gain.

In order to demonstrate that the unlimited-capacity parallel-processing model predicts a difference between the redundancy gains for the fast and slow subsets, we ran several simulations of the model. Of course, there is no clear way of knowing the actual parameters of the input processing times, so we arbitrarily chose the initial parameters of the generating distributions and then varied these parameters systematically. The results, therefore, must be taken only qualitatively.

The first series of simulations was run under the assumption of a Gaussian distribution of input processing times. The parameters of the generating distribution, mu and sigma, were varied systematically. In each variation, 1,000 trials were run in the single-target condition and 1,000 trials in the redundant-target condition. For redundant-target trials, the faster of two samples from the distribution was chosen. The overall means and standard deviations obtained from these simulations are provided in Table 1 . The results show that the magnitude of the overall redundancy gain was dependent on the size of the variance, but, unsurprisingly, had no relation to the mean of the distribution. As we expected, a difference between the redundancy gains for the fast and slow subsets yielded by the randomly-favored-position analysis was observed for all variances used, and was greater for larger variances (Table 2).

Another important aspect of the results is that the variance for the redundant-target trials was consistently smaller than the variance for the single-target trials (Table 1). This finding, of course, directly relates to our prediction that there would be a difference between the
Table 1

Simulations of Performance on Single-Target and Redundant-Target Trials of a Parallel, Unlimited-Capacity Model with Gaussian, Gamma, and Exponentially Distributed Input Processing Times

\begin{tabular}{|c|c|c|c|c|c|c|c|}
\hline \multicolumn{3}{|c|}{ Parameters } & \multicolumn{2}{|c|}{$T=1$} & \multicolumn{2}{|c|}{$\mathrm{T}=2$} & \multirow[b]{2}{*}{ Gaın } \\
\hline $\mathrm{mu}$ & sigma & tau & $M$ & $S D$ & $M$ & $S D$ & \\
\hline \multicolumn{8}{|c|}{ Gaussian } \\
\hline 200 & 28 & & 201 & 28 & 183 & 23 & 8 \\
\hline 200 & 45 & & 202 & 44 & 174 & 37 & 28 \\
\hline 400 & 28 & & 400 & 27 & 384 & 23 & 16 \\
\hline 400 & 45 & & 398 & 46 & 376 & 36 & 22 \\
\hline 400 & 63 & & 398 & 65 & 366 & 53 & 32 \\
\hline 400 & 89 & & 399 & 87 & 353 & 73 & 46 \\
\hline \multicolumn{8}{|c|}{ Gamma } \\
\hline 200 & 28 & & 200 & 28 & 184 & 22 & 16 \\
\hline 200 & 45 & & 201 & 45 & 176 & 33 & 25 \\
\hline 200 & 63 & & 201 & 65 & 163 & 45 & 38 \\
\hline \multicolumn{8}{|c|}{ Exponential } \\
\hline & & 28 & 27 & 27 & 14 & 13 & 13 \\
\hline & & 45 & 41 & 42 & 23 & 21 & 18 \\
\hline
\end{tabular}

Note-Processing times are given in milliseconds. $T=$ number of targets.

sizes of the redundancy gain for the fast and slow subsets in the random-favored-position analysis. On redundanttarget trials, the faster of two input processing times was selected. These faster times could not be faster than the fastest single-target processing times, and only rarely could they be as slow as the slowest single-target processing times. The resultant distribution of the fastest finishing times for redundant targets had a variance that was smaller than that for the distribution of single-target processing times.

Three simulations were also run using a gamma (positively skewed) distribution that generated input processing times with parameters mu and sigma. On the basis of the results with the Gaussian distribution, it was deemed necessary to manipulate only the variance of the distribution. As shown in Tables 1 and 2, increasing the variance of the gamma distribution had effects similar to those of the normal distribution.

Simulations were also run using exponentially distributed input processing times. The parameter tau (w/hich describes both the mean and the standard deviation of the generating distribution) was varied to equal two of the sigma parameters used for the Gaussian and gamma generating distributions. As a result, the mean of the generating distribution was considerably smaller than the means for the Gaussian and gamma distributions. However, this was not of concern since no attempt was being made to approximate the actual parameters of a distribution of input processing times. The exponential distribution simulation produced results, as shown in Tables 1 and 2, qualitatively similar to those of the Gaussian and gamma. distributions.

Interestingly, both van der Heijden et al. (1983) and Mullin and Egeth (in press, Experiment 2) reported statistical equivalence between the redundancy gains for the fast and slow subsets (there were small differences 
Table 2

\begin{tabular}{|c|c|c|c|c|c|c|c|c|}
\hline \multirow{2}{*}{\multicolumn{3}{|c|}{ Parameters }} & \multirow{3}{*}{$\frac{\mathrm{T}=1}{M}$} & \multicolumn{2}{|c|}{ Fast Subsets } & \multicolumn{3}{|c|}{ Slow Subsets } \\
\hline & & & & \multirow{2}{*}{$\begin{array}{c}T=2 \\
M\end{array}$} & \multirow[b]{2}{*}{ Gain } & \multirow{2}{*}{$\frac{\mathrm{T}=1}{M}$} & \multirow{2}{*}{$\frac{T=2}{M}$} & \multirow[b]{2}{*}{ Gain } \\
\hline $\mathrm{mu}$ & sigma & tau & & & & & & \\
\hline \multicolumn{9}{|c|}{ Gaussian } \\
\hline 200 & 28 & & 179 & 164 & 15 & 223 & 202 & 22 \\
\hline 200 & 45 & & 167 & 145 & 22 & 237 & 204 & 33 \\
\hline 400 & 28 & & 378 & 366 & 12 & 421 & 403 & 18 \\
\hline 400 & 45 & & 362 & 348 & 14 & 435 & 405 & 30 \\
\hline 400 & 63 & & 346 & 324 & 22 & 451 & 407 & 44 \\
\hline 400 & 89 & & 331 & 294 & 37 & 468 & 411 & 57 \\
\hline \multicolumn{9}{|c|}{ Gamma } \\
\hline 200 & 28 & & 177 & 167 & 10 & 223 & 201 & 22 \\
\hline 200 & 45 & & 165 & 149 & 16 & 237 & 203 & 34 \\
\hline 200 & 63 & & 151 & 128 & 23 & 251 & 199 & 52 \\
\hline \multicolumn{9}{|c|}{ Exponential } \\
\hline & & 28 & 8 & 4 & 4 & 46 & 23 & 23 \\
\hline & & 45 & 12 & 7 & 5 & 70 & 38 & 32 \\
\hline
\end{tabular}

Note-Processing tumes are given in milliseconds. $\mathrm{T}=$ number of targets.

of a few milliseconds). In light of our simulations, these are obviously perplexing results. Our construal of the unlimited-capacity model in the simulations was, however, rather simple. Other factors might play a role. For example, the processing times for two redundant inputs might not be completely independent due to the variability of a subject's motivational state. Within the overall input processing time distribution, there might be a family of input processing time distributions, each of which corresponds to a particular motivational level of the subject. On a given redundant-target trial, the processing times for the two inputs would be sampled from the distribution corresponding to the motivational state of the subjects for that trial. Since, as we have shown, the magnitude of the redundancy gain is determined by the variance of the distribution of processing times, the net effect of sampling from one of the family of distributions would be to reduce the size of the redundancy gain relative to the gain expected if processing times were sampled from the overall distribution (i.e., the subject had a single motivational state).

The reduction in the overall redundancy gain is reflected in the random-favored-position analysis primarily by a reduction in the redundancy gain for the slow-subset comparison. Thus, if the variance of input processing times given a certain motivational state is small relative to the variance between the distributions for the various motivational states, relatively little difference would be expected between the redundancy gains for the fast and slow subsets when processing capacity is unlimited. Recall that when two redundant targets are processed independently (i.e., a single motivational state), and one input finishes in the slow tail of the distribution of processing times, the other input has a good chance of finishing faster by a relatively large margin. However, if input processing times on a given trial are sampled from one of the distributions corresponding to a particular motivational state, and one of the inputs is processed slowly, the second can only finish faster by a margin that is dependent on the smaller variance of that distribution. The same logic applies for trials where one input is processed quickly, but, in this situation, the probability that a redundant input will finish faster than the first input by a large margin is already constrained by the fact that the first input finished in the fast tail of the distribution of processing times. The introduction of a family of motivational-state distributions with small variances should therefore increase the variance of RTs from redundant-target trials. Thus, if we compare single-target and redundant-target trials, we would observe less of a difference between the variances for the two conditions, and less of a difference between the redundancy gains for the fast and slow subsets yielded by the random-favored-position analysis. The critical factor for obtaining complete equivalence appears to be that the RTs from any one of the motivational-state distributions are not divided between the fast and slow subsets. Thus, it would appear that the unlimited-capacity model can accommodate the result of equivalence between the redundancy gains for the fast and slow subsets.

We have been unable to conceive of a way in which a limited-capacity model could account for the equivalence in the redundancy gains for the fast and slow subsets on the basis of random positional preferences. The effect of the random favored-positions is to induce negative correlations between the redundant-target input processing times. Negative correlations between input processing times have the opposite effect of the positive correlations that occur when a subject's motivational states vary as described above. Thus, these negative correlations increase the variance of the single-target trial RTs, 
and increase the difference between the redundancy gains for the fast and slow subsets. Therefore, the result that the random-favored-position analysis yields equivalence between the redundancy gains for the fast and slow subsets appears to be interpretable as evidence for unlimitedcapacity processing.

A problem that remains is distinguishing unlimitedcapacity models and limited-capacity models with positional preferences when an empirical difference is observed between redundancy gains for the fast and slow subsets. One interesting idea is suggested by manipulations introduced by Biederman and Checkosky (1970) and van der Heijden et al. (1983) to vary the difficulty of discriminating between the target and nontarget stimuli. They argued that decreasing the discriminability between the targets and the nontarget stimuli would increase both the mean and the variance of response latencies. In essence, they attempted to effect a change in the variance of the distribution of input processing times. As we have seen from the simulations, increasing the variance results in a larger overall redundancy gain with parallel, unlimitedcapacity processing.

Unfortunately, limited-capacity models with positional preferences can also predict an increase in the artifactual redundancy gain when the variance of input processing times is increased. When there is no overlap between the distributions of processing times for the favored and unfavored targets, all RTs for redundant-target trials will be determined by the processing time for the target in the favored position. In contrast, recall that if there is some overlap between the distributions of processing times for favored and unfavored targets, the RTs for a certain proportion of the redundant-target trials will be determined by an unfavored target that is processed more quickly than a slowly processed target in the favored position. Increasing the overlap, then, has the effect of reducing the proportion of RTs determined by a slowly processed target in the favored location, and results in a faster mean for redundant-target trials.

The effect of increasing the variance of the distribution of processing times by manipulating the discriminability of the target and nontarget stimuli would be to increase the degree of overlap between the processing times for unfavored and favored targets. Therefore, a larger artifactual redundancy gain would be observed. Similarly, a larger artifactual redundancy gain would be observed if the difference between the mean processing times for the unfavored and favored targets were to increase as a result of the discriminability manipulation.
At this time, we have no suggestions for alternative analyses or manipulations that might be used to determine whether a redundancy gain can be attributed to limitedcapacity processing with randomly varying positional preferences or to unlimited-capacity processing when the redundancy gain for the slow subsets is greater than the redundancy gain for the fast subsets yielded by the random-favored-position analysis. However, given the ability of the redundant-target task to distinguish between the models on the basis of several other empirical outcomes, and given the interpretive difficulties with other techniques, such as examining $\mathrm{RT} /$ display size functions in visual search tasks, we feel that the redundant target detection task deserves further attention.

\section{REFERENCES}

Biederman, I, \& Checkosky, S. F (1970). Processing redundant information. Journal of Experimental Psychology, 83, 486-490

EgeTh, H. E., Folk, C , \& Muluin, P. (in press). Spatial parallehsm in the processing of lines, letters and lexicality. In B. E. Shepp \& $S$ Ballesteros (Eds.), Object perception - Structure and proces. . Hillsdale, NJ: Erlbaum.

ERIKSEN, C. W. (1966). Independence of successive inputs and uncorrelated error in visual form perception. Journal of Experimenial Psychology, 72, 26-35.

HeIJden, A. H. C., van der (1975). Some evidence for an unlimitedcapacity parallel self-terminating process in simple visual search tasks. Acta Psychologica, 39, 21-41

heijden, A. H. C., van der, La Heij, W., \& Boer, J. P. A. 1 1983 ). Parallel processing of redundant targets in simple visual search tasks. Psychological Research, 45, 235-254.

Mullin, P. A., \& EceTh, H. E. (In press). Capactry limitations in visual word processing. Journal of Expenmental Psychology: Human Perception \& Performance.

Santee, J. L., \& Egeth, H. E. (1982). Independence versus interference in the perceptual processing of letters Perception \& Fsychophysics, 31, 101-116.

SNODGRASS, J. G., \& TownSEND, J T (1980) Companng parallel and serial models: Theory and implementation. Journal of Experimental Psychology: Human Perception \& Performance, 6, 330-354.

TownSEND, J. T. (1971). A note on the identifiability of parallel and serial processes. Perception \& Psychophysics, 10, 161-163

TownsEND, J. T. (1972). Some results concerning the identifiability of parallel and serial processes Britlsh Journal of Statistical Psychology, 25, 168-199

TOWNSEND, J. T. (1974) Issues and models concerning the processing of a finite number of inputs. In B. Kantowitz (Ed.), Human information processing: Tutorials in performance and cognition ( $\mathrm{pp} .133$ 185). Hillsdale, NI: Erlbaum.

(Manuscript received November 9, 1987, revision accepted for publication December 3, 1987 ) 